SCIREA Journal of Mathematics

http://www.scirea.org/journal/Mathematics

March 8, 2021

Volume 6, Issue 1, February 2021

SCIREA

\title{
Geneva Mechanism Geometric Resolution
}

\section{Eduardo Martinez Escobedo}

Universidad de Monterrey / Universitat Politècnica de València

Email: eduardo.martinez@udem.edu

\begin{abstract}
Geneva mechanisms have been used to obtain an output intermittent circular motion by the means of a continuous circular motion. It offers the option of having a rest time with between motions in the output link. This paper is intended to provide the geometric resolution of this mechanisms via number of steps and diameter of output link. The approach is theorical and does not include tolerances for pins, grooves or locking wheels.
\end{abstract}

Keywords: geneva, mechanism, geokmetric, resolution

\section{Geneva mechanism definition}

A Geneva mechanism is comprised of two wheels, a driver and a driven.

The driver wheel [DRW] is composed of a cam pin [CP] (left), the blocking wheel [BW] (center) and the rotate indentation [RI] (right). 

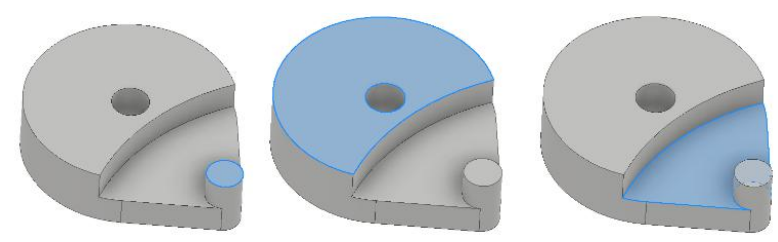

Figure 1: Driver Wheel

The driven wheel (DNW) is composed of grooves [G] (left) and the locking wheel [LW] (right).
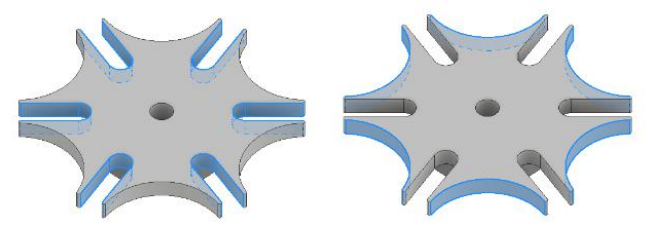

Figure 2: Driven Wheel

The DRW describes a continuous circular motion while the DNW will describe a circular intermittent motion in angular steps equivalent to $360^{\circ}$ divided by the number of $\mathrm{G}$. The direction of the motion in the DNW is inverted in relation to the DRW.
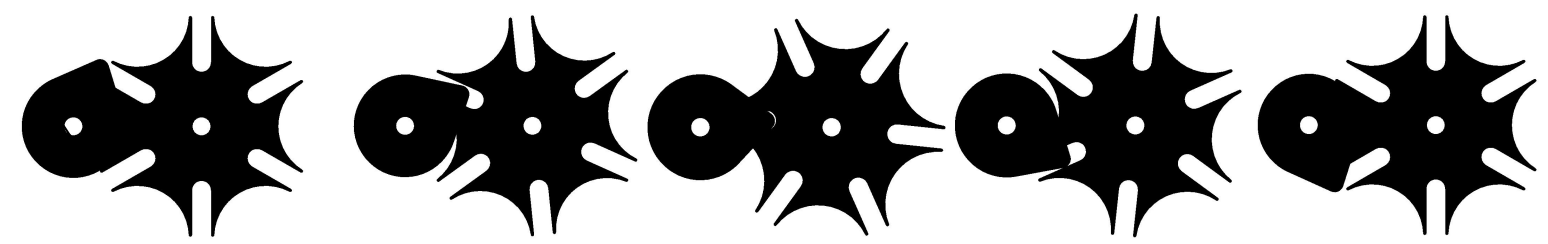

Figure 3: Geneva Drive

The DNW's motion is blocked when CP is not inside any of $\mathrm{G}$ by means of the interaction between the BW and the LW.

For the DNW to turn $360^{\circ}$, the DRW shall turn G times.

\section{Nomenclature}

$\begin{array}{llll}\text { DRW } & \text { Driver Wheel } & \text { RI } & \text { Rotational Indentation } \\ \text { CP } & \text { Cam Pin } & \text { DNW } & \text { Driven Wheel } \\ \text { BW } & \text { Blocking Wheel } & \text { ND } & \text { Driven Wheel outside diameter }\end{array}$


G Grooves

LW Locking Wheel

AG Angle Between Grooves

CPC Cam Pin Pitch circle
HAG Half the Angle Between Grooves

GD Grooves dept

P Cam Pin Diameter

\section{Geometric resolution}

Given the first input value DNW outside diameter [ND] and the angle between G [AG] which is equal to $360^{\circ}$ divided by the number of G; the construction of the DRW and DNW as follows:

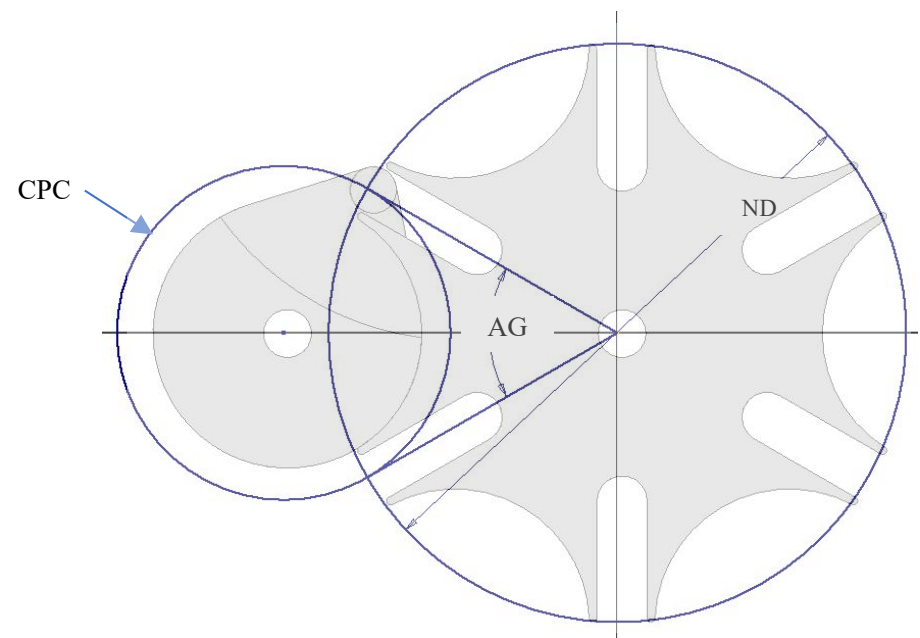

Figure 4: Basic construction

The lines describing the AG shall origin from the center of the DNW and end in the circumference of DNW. The geometry described in the left circle is the CP pitch circle [CPC] shown in Figure 5: Cam Pin Pitch Circle.

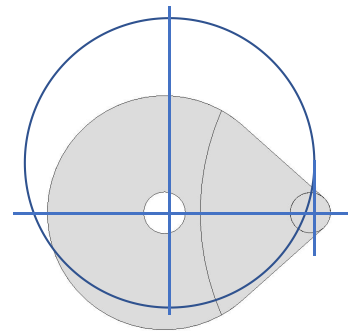

Figure 5: Cam Pin Pitch Circle 
AG lines must be tangent to CPC. The end of the lines that describe AG in contact with the circumference with DNW must also be coincident with the circumference of CPC. If the two centers are aligned to the horizontal axis, a condition that fully constrains the geometry will be present.

The center distance between DRW and DNW is described the hypothenuse of the right triangle conformed from the points A, B and C as seen in Figure 6: Center Distance between DRW \& DNW.

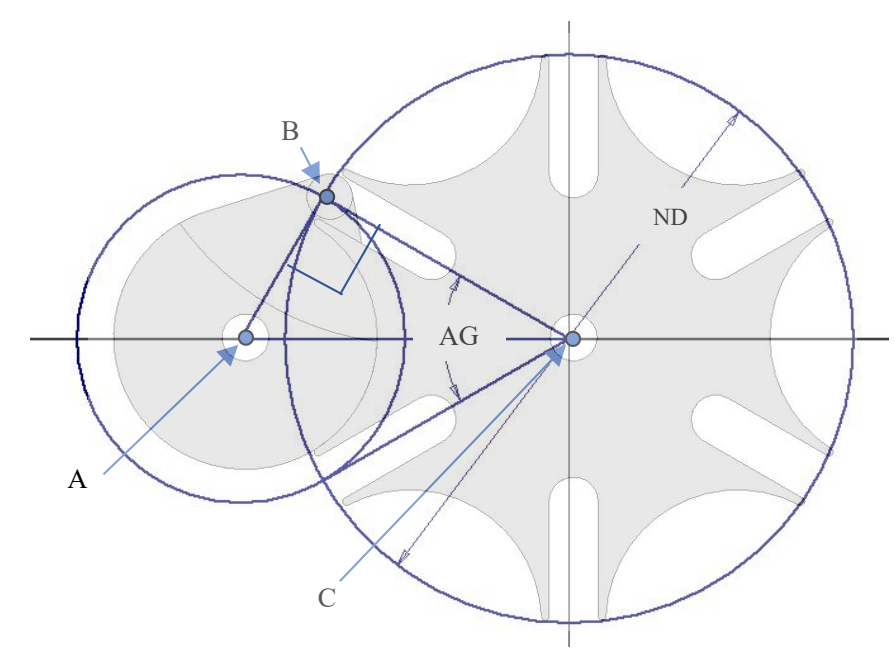

Figure 6: Center Distance between DRW \& DNW

" $A$ " is the center of the DRW, "B" is the intersection of a line from the center of DRW to the circumference of CPC and the line from the center of DNW to the circumference of DNW tangent to CPC. Point " $\mathrm{C}$ " is the center of DNW. The angle between lines "AB" and "AC" is half $\mathrm{AG}$ [HAG].

Diameter CPC can be calculated by:

$$
C P C=N D \times \tan H A G
$$

Equation 1: Cam Pin Pitch Diameter

Lines "AB" and "BC" form a right angle, thus the center distance between DRW and DNW [WCD] can be described as: 


$$
W C D=\frac{N D}{2 \cos H A G}
$$

Equation 2: Wheels Center Distance

Groove depth [GD] is the distance from the circumference of DNW to the circumference concentric to DNW and tangent to DRW.

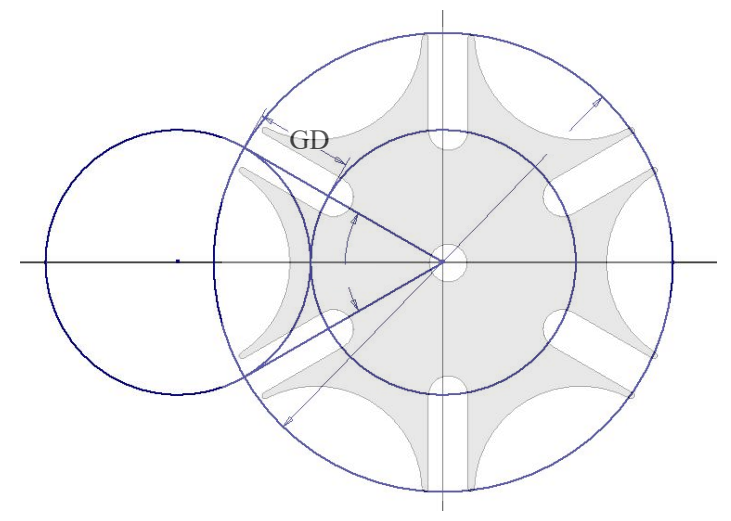

Figure 7: Groove Depth

GD can be expressed as:

$$
G D=\frac{D N W+C P C}{2}-W C D
$$

Equation 3: Groove Depth

The locking wheel (which is equivalent for the blocking wheel) from the driven wheel can be calculated by the means of the Cam Pin diameter $[\mathrm{P}]$ and is concentric to the DRW. Locking wheel will be:

$$
L W=C P C-1.5 P
$$

Equation 4: Locking Wheel Diameter 


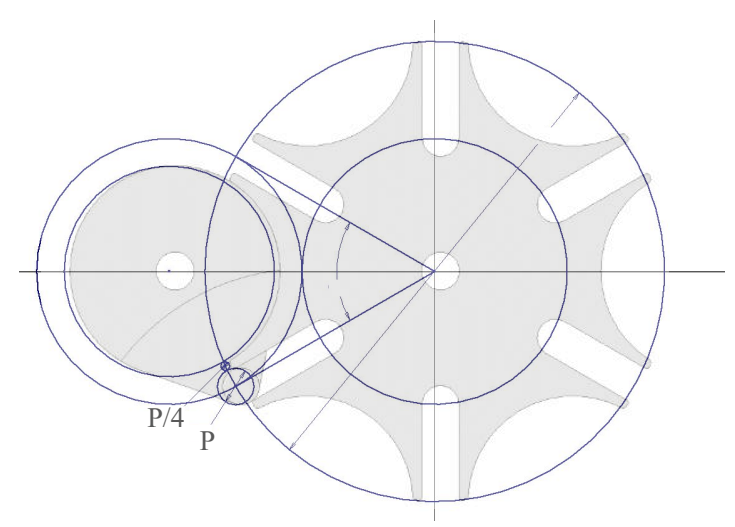

Figure 8: Locking Wheel

The Rotation Indentation diameter is concentric with the DNW and can be defined as:

$$
R I=D N W+(P / 2)
$$

Equation 5: Rotation Indentation Diameter

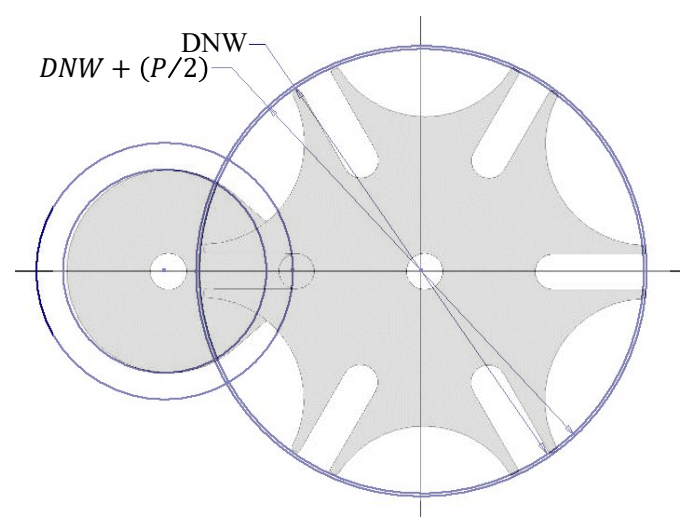

Figure 9: Rotation Indentation

\section{Conclusions}

Geneva mechanism has a simple construction and can offer a solution for indexing tables, or any mechanism with intermittent circular motion. Thru the years it has proven to be inexpensive and durable. Serve this document as a guide to the geometric dimensioning of the mechanism. 


\section{References}

[1] Brickford, J. (1964, December). Mechanisms for intermittent motion. Machine Design, 37, 121-131.

[2] Hasty, C. E., \& Potts, J. F. (1966). Analysis and Synthesis Procedures for Geneva Mechanism Design. IBM Journal of Research and Development, 10(3), 186-197. https://doi.org/10.1147/rd.103.0186

[3] Hunt, K. H., Fink, N., \& Nayar, J. (1960). Linkage Geneva Mechanisms. A Design Study in Mechanism Geometry. Proceedings of the Institution of Mechanical Engineers, 174(1), 643-668. https://doi.org/10.1243/PIME_PROC_1960_174_051_02

[4] Jones, F. D. (Ed.). (1930). Intermittent Motions from Gears and Cams. In Ingenious Mechanisms for Designers and Inventors (Vol. 1, pp. 74-76). New York, NY: Industrial Press. 